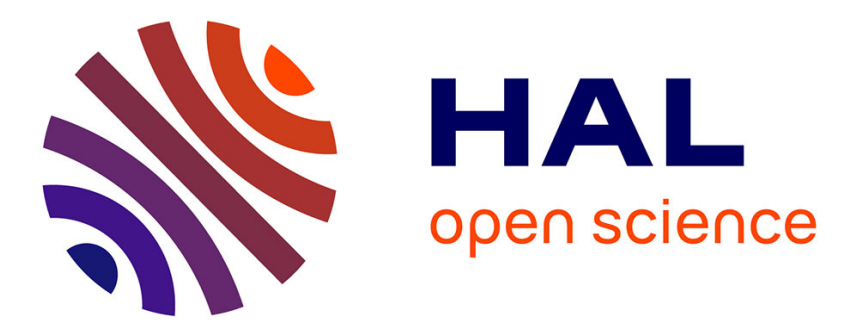

\title{
Questioning the relationship between the $\chi 4$ susceptibility and the dynamical correlation length in a glass former
}

Rémy Colin, Ahmed M. Alsayed, Cyprien Gay, Bérengère Abou

\section{- To cite this version:}

Rémy Colin, Ahmed M. Alsayed, Cyprien Gay, Bérengère Abou. Questioning the relationship between the $\chi 4$ susceptibility and the dynamical correlation length in a glass former. Soft Matter, 2015. hal$01098350 \mathrm{v} 3$

\section{HAL Id: hal-01098350 \\ https://hal.science/hal-01098350v3}

Submitted on 10 Sep 2015 (v3), last revised 19 Mar 2021 (v4)

HAL is a multi-disciplinary open access archive for the deposit and dissemination of scientific research documents, whether they are published or not. The documents may come from teaching and research institutions in France or abroad, or from public or private research centers.
L'archive ouverte pluridisciplinaire HAL, est destinée au dépôt et à la diffusion de documents scientifiques de niveau recherche, publiés ou non, émanant des établissements d'enseignement et de recherche français ou étrangers, des laboratoires publics ou privés. 


\title{
Questioning the relationship between the $\chi_{4}$ susceptibility and the dynamical correlation length in a glass former
}

\author{
Rémy Colin ${ }^{* a}$, Ahmed M. Alsayed ${ }^{b}$, Cyprien Gay ${ }^{a}$ and Bérengère Abou *a \\ a Laboratoire Matière et Systèmes Complexes, UMR CNRS $7057 \&$ Université Paris Diderot, \\ 10 rue A. Domon et L. Duquet, 75205 Paris Cedex 13, France. \\ E-mail: remy.colin@univ-paris-diderot.fr; berengere.abou@univ-paris-diderot.fr; \\ ${ }^{b}$ Complex Assemblies of Soft Matter Laboratory, UMI CNRS 3254, \\ Rhodia INC., 350 G. Patterson Blvd, Bristol PA 1900\%, USA.
}

(Dated: September 10, 2015)

\begin{abstract}
Clusters of fast and slow correlated particles, identified as dynamical heterogeneities (DHs), constitute a central aspect of glassy dynamics. A key ingredient of the glass transition scenario is a significant increase of the cluster size $\xi_{4}$ as the transition is approached. In need of easy-to-compute tools to measure $\xi_{4}$, the dynamical susceptibility $\chi_{4}$ was introduced recently, and used in various experimental works to probe DHs. Here, we investigate DHs in dense microgel suspensions using image correlation analysis, and compute both $\chi_{4}$ and the four-point correlation function $G_{4}$. The spatial decrease of $G_{4}$ provides a direct access to $\xi_{4}$, which is found to grow significantly with increasing volume fraction. However, this increase is not captured by $\chi_{4}$. We show that the assumptions that validate the connection between $\chi_{4}$ and $\xi_{4}$ are not fulfilled in our experiments.
\end{abstract}

The present version was accepted for publication in Soft Matter

PACS numbers: 05.40.-a, 05.20.-y, 05.70.-a

\section{Introduction}

Understanding the glass transition and the out-ofequilibrium glassy dynamics remains a challenge in condensed matter physics. In practice, glass transitions are observed in various systems, such as molecular liquids, colloids or granular materials 1-3. Among all, dense colloids are model systems with a glass transition at ambient temperature upon increasing volume fraction [4. They display slow but accessible timescales and can be probed with simple optical techniques such as microscopy and dynamic light scattering 5, 6.

Over the last 15 years, dynamical heterogeneities (DHs) have been recognized as a promising feature in understanding slow relaxation processes in glass-forming systems $7-9$. DHs consist of fast and slow clusters of dynamically correlated particles, coexisting in the material, with the idea that a dynamical correlation length - representing the clusters size - diverges when approaching the glass transition 10-16. Dynamical heterogeneities are predicted by theories and have been observed in numerical simulations [11, 17, 23, and experimental works 2432 . They can mainly be quantified with tools such as four-point correlation functions $G_{4}$, whose spatial dependence gives a direct access to the dynamical correlation length $\xi_{4}$, or with dynamical susceptibilities $\chi_{4}$ which have recently been proposed as easy-to-compute indirect tools [11, 24.

Here, we investigate DHs with both a four-point correlation function $G_{4}$ and its associated dynamical sus-

*R.C.'s current address: Max Planck Institute for Terrestrial Microbiology, Karl-von-Frisch Strasse 16, 35043 Marburg, Germany ceptiblity $\chi_{4}$, in dense suspensions of soft microgel particles, by performing image correlation analysis. With the direct tool $G_{4}$, we measure a significant growth of the dynamical correlation length $\xi_{4}$ with increasing volume fraction. We then investigate the validity of the dynamical susceptibility $\chi_{4}$ as a tool to extract the dynamical correlation length $\xi_{4}$, and analyze the reasons why it fails to quantify the growth of $\xi_{4}$.

\section{THEORETICAL TOOLS FOR MEASURING THE CORRELATION LENGTH}

We consider a system described by a local order parameter $q_{\mathbf{j}, t}(\tau)$, here defined as the time correlation of the observable quantity (e.g. local density, particle position, transmitted light intensity) between time $t$ and $t+\tau$ at point $\mathbf{j}$. A space-averaged and a space-time-averaged order parameters, $Q_{t}(\tau)=\left\langle q_{\mathbf{j}, t}(\tau)\right\rangle_{\mathbf{j}}$ and $Q(\tau)=\left\langle Q_{t}(\tau)\right\rangle_{t}$ respectively, are constructed so that the averaged timecorrelation function $Q(\tau)$ measures the relaxation dynamics of the system. This quantity decays from 1 to 0 as particles move a characteristic distance between $t$ and $t+\tau[33$.

A direct route to the correlation length $\xi_{4}$ is the 4point correlator $G_{4}^{\text {vect }}(\mathbf{r}, \tau)$ which reflects how $q_{\mathbf{j}, t}(\tau)$ is correlated between points separated by $\mathbf{r}$ :

$$
\begin{aligned}
G_{4}^{\mathrm{vect}}(\mathbf{r}, \tau) & =\left\langle\left(q_{\mathbf{j}+\mathbf{r}, t}(\tau)-q_{\mathbf{j}+\mathbf{r}}(\tau)\right)\left(q_{\mathbf{j}, t}(\tau)-q_{\mathbf{j}}(\tau)\right)\right\rangle_{\mathbf{j}, t} \\
& =\left\langle q_{\mathbf{j}+\mathbf{r}, t}(\tau) q_{\mathbf{j}, t}(\tau)\right\rangle_{\mathbf{j}, t}-\left\langle q_{\mathbf{j}+\mathbf{r}}(\tau) q_{\mathbf{j}}(\tau)\right\rangle_{\mathbf{j}}
\end{aligned}
$$

where $q_{\mathbf{j}}(\tau)=\left\langle q_{\mathbf{j}, t}(\tau)\right\rangle_{t}$. The corresponding 4-point correlation function $G_{4}(r, \tau)$ is defined as, $G_{4}(r, \tau)=$ $\left\langle G_{4}^{\text {vect }}(\mathbf{r}, \tau)\right\rangle_{r<|\mathbf{r}|<r+\delta r}$, with $\delta r$ chosen such that the average runs over a sufficient number of points. In a system 
with a dominant dynamical correlation length scale $\xi_{4}(\tau)$, the correlation function decays at large $r$ as:

$$
G_{4}(r, \tau) \sim \frac{1}{r^{p}} \exp \left(-r / \xi_{4}(\tau)\right)
$$

where exponent $p$ is discussed below. Equation (2) defines the dynamical correlation length $\xi_{4}$.

Another tool was introduced recently to characterize dynamical heterogeneities, namely the dynamical susceptibility $\chi_{4}(\tau)$ (see [18, 33, for a review). It can be measured as the variance of the temporal fluctuations of $Q_{t}(\tau)$

$$
\chi_{4}(\tau)=N\left(\left\langle Q_{t}^{2}(\tau)\right\rangle_{t}-\left\langle Q_{t}(\tau)\right\rangle_{t}^{2}\right)
$$

with $N$ the number of points in space under consideration. Let us see how $\chi_{4}$ is indirectly connected to $\xi_{4}$. Since $Q_{t}(\tau) \equiv\left\langle q_{\mathbf{j}, t}(\tau)\right\rangle_{\mathbf{j}}$, it can be shown that $\chi_{4}$ is related to $G_{4}$ by $\chi_{4}(\tau)=\sum_{\mathbf{r}} G_{4}^{\text {vect }}(\mathbf{r}, \tau)$ which can be expressed in the continuous limit as :

$$
\chi_{4}(\tau)=\rho \int \mathrm{d}^{2} \mathbf{r} G_{4}(|\mathbf{r}|, \tau)
$$

with $\rho$ the average density of points in space. It was recently proposed to use $G_{4}(r, \tau) \sim$ $A(\tau) / r^{p} \exp \left(-r / \xi_{4}(\tau)\right)$ and Eq. (4) to clarify the link between $\chi_{4}(\tau)$ and $\xi_{4}(\tau)$. In two dimensions, $\chi_{4}(\tau)$ is then :

$$
\chi_{4}(\tau) \sim A(\tau) 2 \pi \rho \xi_{4}^{2-p}(\tau)
$$

The peak of $\chi_{4}(\tau)$ was proposed and used widely in numerical simulations and experiments $24,25,27,34,35$. to determine the time for which the dynamics is the most heterogeneous and indirectly, the correlation length value $\xi_{4}$. Here, the exponent $p$ can be related to the clusters fractal dimension or, if the clusters are all compact, to the cluster size distribution. As stated in [18, the growing peak in $\chi_{4}$ upon increasing volume fraction reveals the growth of a dynamical correlation length if the assumptions made for the scaling of $G_{4}$ are fulfilled.

\section{MATERIALS AND METHODS}

Microgel suspension preparation. Our model glass consists of a suspension of thermosensitive microgels, made of pNIPAm (poly-N-isopropylacrylamide) crosslinked with BIS (N,N'-methylenebisacrylamide), whose synthesis is described in 14]. The microgel diameter decreases with temperature, which provides a unique way of tuning volume fraction with an external parameter [36, 37. The suspension was prepared by mixing small and large microgels with a diameter ratio $1: 1.8$, constant over the investigated temperature range $20-30^{\circ} \mathrm{C}$ (number fraction of large particles : $18 \%$ ). The bidispersity was used to suppress crystallisation. Figure
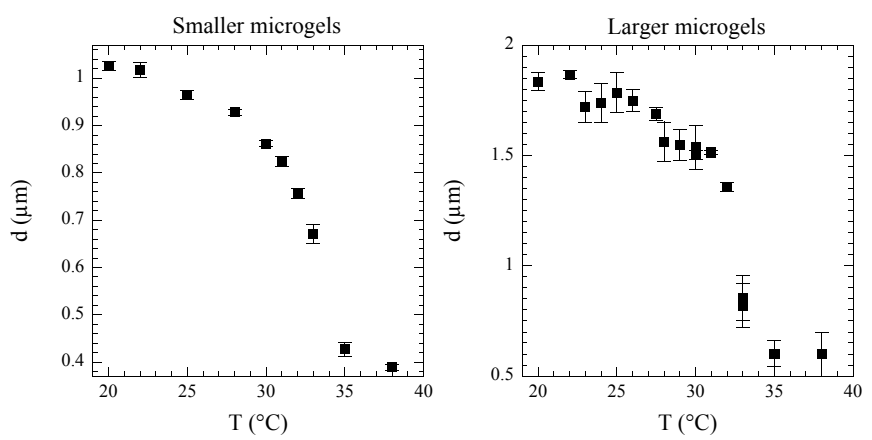

FIG. 1: Microgels diameter as a function of temperature, measured with Dynamic Light Scattering. In order to suppress crystallisation, our suspension is prepared as a bidisperse mixture of small microgels (left) and large ones (right) (the number fraction of large particles is $18 \%$ ). The microgels diameter decreases over the investigated temperature range $\left(20-30{ }^{\circ} \mathrm{C}\right)$, with a constant diameter ratio 1:1.8.

1 shows the microgel diameters as a function of temperature, measured with Dynamic Light Scattering. The diameters decrease by $\sim 20 \%$ over the investigated temperature range. Within the $20-30{ }^{\circ} \mathrm{C}$ range, the pNIPAm particles behave as hydrophilic repulsive soft spheres (the transition to hydrophobic attractive spheres occurs at $32-33^{\circ} \mathrm{C}$ and is easily identified by microscopy).

Effective volume fraction of the suspension. At temperature $\mathrm{T}=30^{\circ} \mathrm{C}$, an effective volume fraction was assigned to the microgel suspension. For this purpose, latex probes (radius $R=0.5 \mu \mathrm{m}$ comparable to the microgel radius) were added to the suspension and their mean-squared displacement (MSD) measured. The MSD was found to increase linearly with time, which defines a diffusion coefficient $D,\left\langle\Delta r^{2}(\tau)\right\rangle_{i, t}=4 D \tau$. A suspension viscosity $\eta=45 \mathrm{mPa}$.s was deduced from the StokesEinstein relation, $\eta=k_{B} \mathrm{~T} / 6 \pi R D$, expected to be valid at such low viscosity. The main issue with using the Stokes- Einstein relation in this case is that the size of the probe is similar to the size of the pNIPAm particle. This issue is tackled by considering the long time diffusion coefficient which shows the response to the medium on larger length scales. Following previous works performed in pNIPAm suspensions similar to ours 38], the suspension volume fraction was estimated from the measured value $\eta / \eta_{\text {solvent }}$, using the empirical expression given in this paper. In our case, the volume fraction at $T=30^{\circ} \mathrm{C}$ was approximately $\Phi_{30}=0.49$. The volume fractions at lower temperatures $T$ are then calculated using the relation $\Phi_{\text {eff }}(\mathrm{T})=\left(\bar{d}(\mathrm{~T}) / \bar{d}\left(30^{\circ} \mathrm{C}\right)\right)^{3} \Phi_{30}$, where $\bar{d}(T)$ is the number-averaged diameter $0.82 d_{\text {small }}+0.18 d_{\text {large }}$ at temperature $T$.

Sample preparation and video recording. The microgel suspension was injected in a $3 \times 3 \mathrm{~mm}^{2}$ chamber made of a microscope plate and a coverslip separated by a $250 \mu \mathrm{m}$ thick adhesive spacer. The chamber was sealed with araldite glue to avoid evaporation and contamination. The samples were observed using standard 


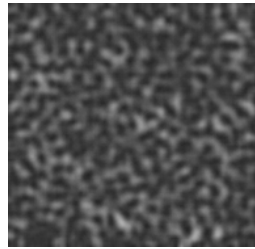

0.49
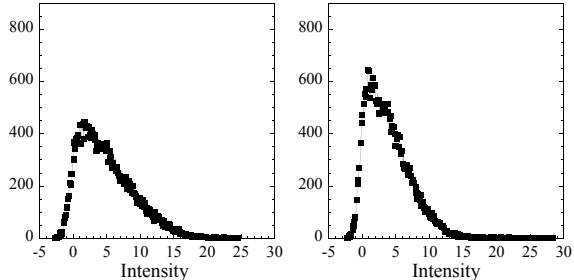

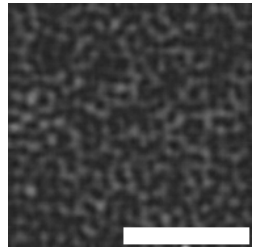

0.60

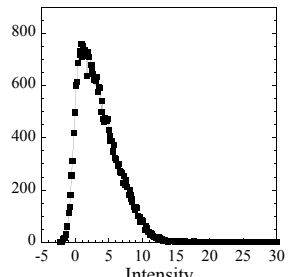

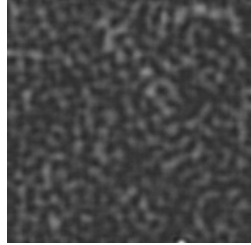

0.64

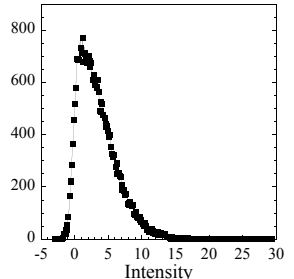

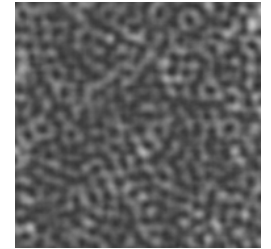

0.66

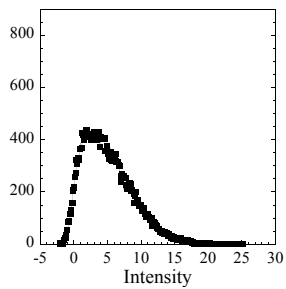

FIG. 2: Images of the microgel suspension at various volume fractions, $(0.49,0.55,0.60,0.64$ and 0.66$)$ in bright field microscopy at $\times 100$ magnification (oil immersion objective, $\mathrm{NA}=1.3)$; the subsets dimension is $187 \times 187 \mathrm{pixel}^{2}$. The white bar is 96 pixel long $(7 \mu \mathrm{m})$. Images have been processed (see details in the appendix) to filter out the noise before image correlation analysis. The histogram of pixel intensities of these processed images is shown. The condenser aperture stop was set to the same setting for each experiment (condenser $\mathrm{NA}=0.55$ ).

bright field microscopy on a inverted Leica DM IRB microscope at $\times 100$ magnification (oil immersion objective, $\mathrm{NA}=1.3$, depth of focus: $\sim 200 \mathrm{~nm}$ ). Typical images of the suspension at various volume fractions can be seen in Figure 2. The objective temperature was adjusted with a Bioptechs objective heater within $\pm 0.1^{\circ} \mathrm{C}$. The sample temperature was maintained through the immersion oil in contact. A CCD camera (FOculus 124B) coupled to the microscope, was recording films of the microgel suspension. The camera was running at a frame rate from 30 down to $0.375 \mathrm{fps}$ for a few minutes to several hours, depending on the suspension dynamics. The region of observation was chosen at least $100 \mu \mathrm{m}$ away from the sample edges to avoid boundary effects.

Image correlation analysis. Films of the microgel suspension were analyzed with image correlation analy- sis, a suitable technique when particle trajectories cannot be resolved individually. Films consist of successive video frames $\left(640 \times 480\right.$ pixel $\left.^{2}\right)$. Frames are first pre-processed following a procedure described in appendix. Image correlation analysis was then performed on $187 \times 187$ pixel $^{2}$ subsets of the pre-processed frames using home-made ImageJ [39] plugin (subset dimension: $13.6 \times 13.6 \mu \mathrm{m}^{2}, 1$ $\mathrm{px}=72.75 \mathrm{~nm})$. Each frame subset is described by a matrix of pixels $\mathbf{p}$ of intensity $I_{\mathbf{p}}(t)$, where $\mathbf{p}$ is the twodimensional index of the pixel position and $t$ the time position of the frame in the film. Possible global variations of illumination and contrast along the film were wiped out using the normalised frame intensity $i_{\mathbf{p}}(t)$ at pixel p:

$$
i_{\mathbf{p}}(t)=\frac{I_{\mathbf{p}}(t)-\left\langle I_{\mathbf{p}}(t)\right\rangle_{\mathbf{p}}}{\sqrt{\left\langle\left(\delta I_{\mathbf{p}}(t)\right)^{2}\right\rangle_{\mathbf{p}}}}
$$

with $\delta I_{\mathbf{p}}(t)=I_{\mathbf{p}}(t)-\left\langle I_{\mathbf{p}}(t)\right\rangle_{\mathbf{p}}$, and $\langle\cdot\rangle_{\mathbf{p}}$ the average over all the pixels in the frame. The subsets are divided into non-overlapping squared Regions Of Interest, ROI, of dimension equivalent to a particle size, $11 \times 11$ pixel $^{2}(289$ ROIs in a subset). The subset intensity $i_{\mathbf{p}}(t)$ is now denoted as $i_{\mathbf{j}, \mathbf{j}^{\prime}}(t) \equiv i_{\mathbf{p}}(t)$, with $\mathbf{j}$ the center of ROI $[\mathbf{j}]$ and $\mathbf{j}^{\prime} \in \operatorname{ROI}[\mathbf{j}]$. (ROI $[\mathbf{j}]$ is centered around pixel $\mathbf{j}$. ) For each subset, following [40, a local order parameter, $q_{\mathbf{j}, t}(\tau)$, was defined as,

$$
q_{\mathbf{j}, t}(\tau)=\left\langle i_{\mathbf{j}, \mathbf{j}^{\prime}}(t+\tau) i_{\mathbf{j}, \mathbf{j}^{\prime}}(t)\right\rangle_{\mathbf{j}^{\prime} \in R O I[\mathbf{j}]},
$$

The functions $Q(\tau)=\left\langle q_{\mathbf{j}, t}(\tau)\right\rangle_{\mathbf{j}, t}, \chi_{4}(\tau)$ and $G_{4}(r, \tau)$ were then derived from $q_{\mathbf{j}, t}(\tau)$ using our home-made ImageJ plugin.

\section{DYNAMICAL SUSCEPTIBILITY $\chi_{4}$}

The microgel suspension was investigated at various volume fractions in the supercooled states, at volume fractions below the glass transition 14. The volume fraction was increased in a quasistatic way, allowing the system to relax between each step and reach an equilibrium state. Figure 3 a shows the ensemble-averaged order parameter $Q(\tau)=\left\langle q_{\mathbf{j}, t}(\tau)\right\rangle_{\mathbf{j}, t}$, similar to the one in [25, 40, 41] in the microgel suspension at various volume fractions. It decreases from its maximal value - ideally 1 at $\tau=0$ - to 0 at the largest lag times, with a typical decay time $\tau^{\star}$. It is intuitive that $\tau^{\star}$ is related to the particles dynamics : as the particles get farther from their initial positions with increasing lag time $\tau$, the time correlation function $Q(\tau)$ between two frames separated by $\tau$ gets smaller on average. With increasing volume fraction, the relaxation time $\tau^{\star}$, defined by $Q\left(\tau^{\star}\right)=0.25$ [4], increases by three orders of magnitude, revealing the suspension dynamics slowing down.

The dynamical susceptibility $\chi_{4}(\tau)$ is shown in Figure 3-b. It exhibits a maximum whose value increases with volume fraction. In the inset, $\chi_{4}(\tau)$ is plotted as a function of $Q(\tau)$. Its maximum is reached for approximately 

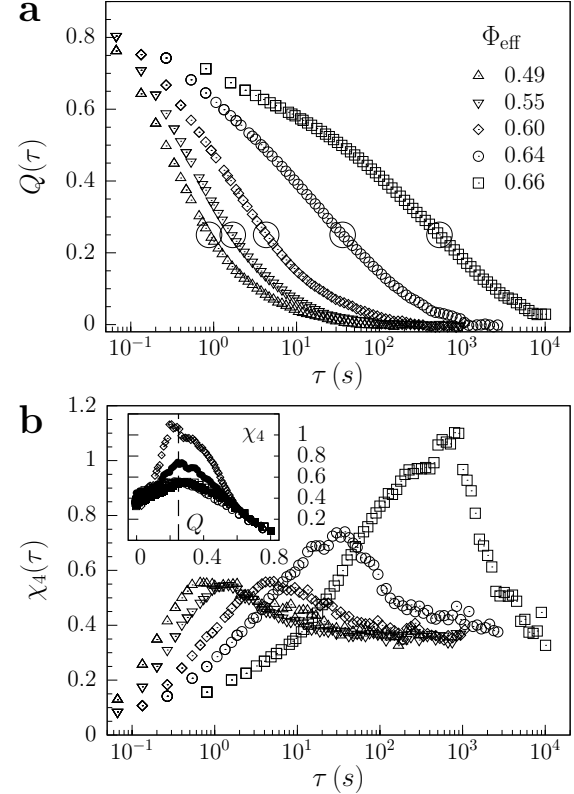

FIG. 3: Mean correlation function $Q(\tau)$ and dynamical susceptibility $\chi_{4}(\tau)$ measured in the microgel suspension with increasing volume fraction. In (a), the values of the typical decay time $\tau^{\star}$ of $Q(\tau)$, denoted by large empty circles, are: $0.88,1.7,4.3,36$ and $5.310^{2} \mathrm{~s}$. In (b), the peak value of $\chi_{4}$ increases with volume fraction, suggesting the increase of a spatial correlation length. The inset reveals that the peak value is reached approximately for $Q(\tau)=0.25$ (vertical line), the value chosen to estimate the typical decay time $\tau^{\star}$.

the same value of $Q(\tau)$, corresponding to the suspension relaxation time $\tau^{\star}$. Under the assumptions detailed in [18, the increase of the peak value $\chi_{4}\left(\tau^{\star}\right)$ with volume fraction suggests an increase of the spatial correlation length $\xi_{4}\left(\tau^{\star}\right)$ with volume fraction, see Eq. (5). In the following, we measure $\xi_{4}\left(\tau^{\star}\right)$ from $G_{4}\left(r, \tau^{\star}\right)$ and test the reliability of Eq. (5).

\section{DIRECT MEASUREMENT OF THE SPATIAL CORRELATION LENGTH $\xi_{4}$}

Let us first focus on the direct measurement of dynamical heterogeneities with a normalised 4-point correlation function $G_{4}(r, \tau) / G_{4}(0, \tau)$ [1], 19. Figure 4 a shows $G_{4}\left(r, \tau^{\star}\right) / G_{4}\left(0, \tau^{\star}\right)$ in the microgel suspension with increasing volume fraction at time $\tau^{\star}$, consistent with the peak value of $\chi_{4}$ (see inset of Figure 4 -b), where dynamical heterogeneities are expected to be at their maximum. At low volume fraction, $G_{4}\left(r, \tau^{\star}\right) / G_{4}\left(0, \tau^{\star}\right)$ exhibits an exponential decay on a length scale $a=0.31 \mu \mathrm{m}$ which we will hereafter name "effective particle radius" as it corresponds approximately to the radius of the most abundant microgel $0.42 \mu \mathrm{m}$ at $T=30^{\circ} \mathrm{C}$. For the two highest volume fractions $\Phi_{\text {eff }}=0.64$ and $\Phi_{\text {eff }}=0.66$, the same exponential decay at small $r$ is followed by a second decay at large $r$. This decay becomes weaker with increas-
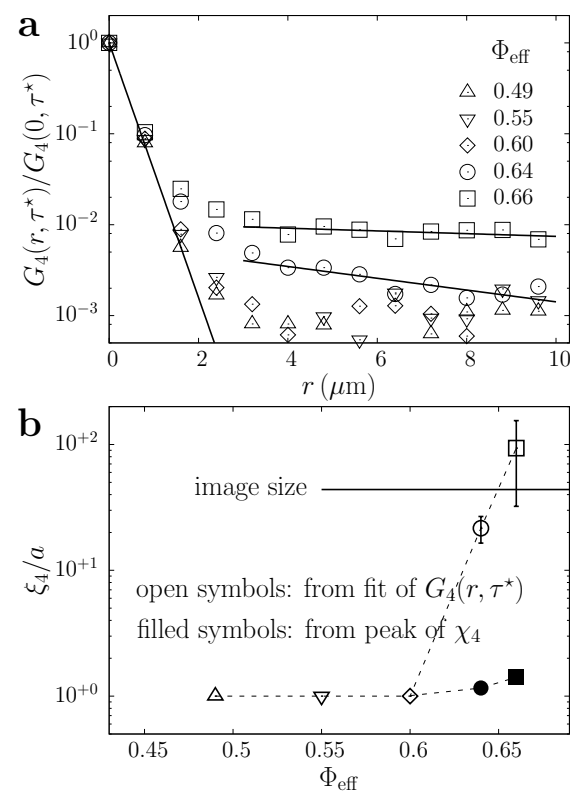

FIG. 4: Normalised 4-point correlation function $G_{4}\left(r, \tau^{\star}\right) / G_{4}\left(0, \tau^{\star}\right)$ and correlation length $\xi_{4}\left(\tau^{\star}\right)$. (a): We define the critical radius $r_{c}$ as the intersection of both asymptotes. The solid lines correspond to fits according to Eq. (8) with $p=0$. (b): The correlation length $\xi_{4}$ extracted from the fits of $G_{4}$ (open symbols) increases significantly with volume fraction. The highest value of $\xi_{4}$ is larger than the image size (horizontal line) and must be considered with caution. Values: $\xi_{4} / a=93 \pm 63$ at $\Phi_{\text {eff }}=0.66 ; \xi_{4} / a=22 \pm 5$ at $\Phi_{\text {eff }}=0.64$; At $\Phi_{\text {eff }}=0.60,0.55$ and 0.49 , the second decay cannot be quantified and we identify the correlation length with the effective particle radius, $\xi_{4}=a$. The values of the correlation length derived from the peak value of $\chi_{4}$ (filled symbols) are significantly lower.

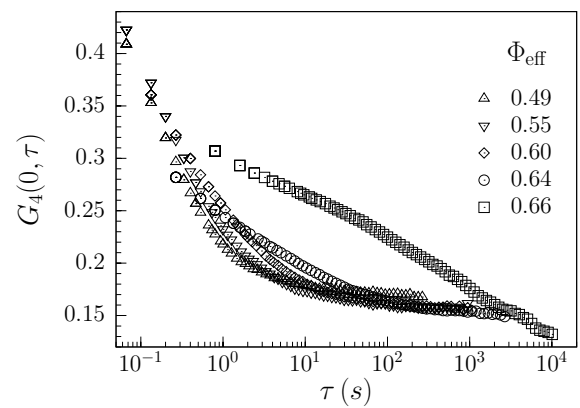

FIG. 5: Prefactor $G_{4}(0, \tau)$ in the microgel suspension as volume fraction increases. It decreases substantially with the lag time, and is found to depend on volume fraction in the microgel suspension.

ing volume fraction, directly suggesting the increase of spatial correlations as the glass transition is approached. The normalised spatial correlation then reaches the noise floor, which is around $10^{-3}$ for all volume fractions.

The high volume fraction data in Figure 4 a for $G_{4}(r)$ were fit in the range $r=3-10 \mu \mathrm{m}$, corresponding to the large $r$-regime, with the following family of functions, 
following Eq. (2):

$$
G_{4}\left(\mathrm{r}, \tau^{\star}\right) / G_{4}\left(0, \tau^{\star}\right) \propto K_{\Phi}\left(\frac{a}{r}\right)^{p} \exp \left(-\frac{r}{\xi_{4}\left(\tau^{\star}\right)}\right)
$$

where the exponent $p$, the correlation length $\xi_{4}$, and the coefficient $K_{\Phi}$ are adjustable parameters [50]. Values of $p$ close to zero were found to provide the best adjustments and we set $p=0$. In order to check the robustness of the $\xi_{4}$ values obtained from the $G_{4}$ measurements, we added extra noise to the film images purposefully, and it showed that the measurement of $\xi_{4}$ is fairly robust against noise. 51

The correlation length $\xi_{4}\left(\tau^{\star}\right)$, shown in Figure 4 -b and extracted from the fit, increases significantly with volume fraction, and reaches a value $(29 \pm 19$ microns $)$ that exceeds the size of the observed field $(13.6 \times 13.6$ microns $)$ for the highest volume fraction investigated.

\section{BAD ESTIMATE OF THE SPATIAL CORRELATION LENGTH FROM THE SUSCEPTIBILITY $\chi_{4}$}

Let us estimate the growth of the correlation length that would be derived from $\chi_{4}$ if one were to use $2 \pi \xi_{4}^{2}\left(\tau^{\star}\right) A=\chi_{4}\left(\tau^{\star}\right)$. At low volume fraction $\Phi_{\text {eff }}=$ $0.49,0.55$ and 0.6 , the peak value of $\chi_{4}$ is a constant. Since no correlations are expected, the correlation length is set equal to the effective particle radius, $\xi_{4}=a$. This also sets the value of the prefactor $A$. At higher volume fraction, keeping the same value $A$ for the whole set of data yields $\xi_{4}^{\Phi_{\text {eff }}=0.64}=1.16 a$ and $\xi_{4}^{\Phi_{\text {eff }}=0.66}=1.41 a$. The growth of this estimate of $\xi_{4}$ is widely underestimated as compared to the direct measurement (see Fig. 4 -b).

Given our experimental data for $G_{4}$, let us investigate the possible sources of error and assess how reliably the growth of $\xi_{4}$ with volume fraction can be inferred from the growing peak in $\chi_{4}$. Since $\chi_{4}$ and $G_{4}$ are related through Eq. (4), let us investigate $G_{4}$ in more detail. As shown in Fig. $4 \mathrm{a}, G_{4}$ displays two spatial regimes for the highest volume fractions, with a crossover radius $r_{c}$ corresponding to approximately 5 effective particle radii $\left(a=0.31 \mu \mathrm{m}, r_{c}=1.4 \mu \mathrm{m}\right.$ for $\Phi_{\text {eff }}=0.66$ and $r_{c}=1.7 \mu \mathrm{m}$ for $\left.\Phi_{\text {eff }}=0.64\right)$. We now estimate both parts of the integral in Eq. (4), $r<r_{c}$ and $r>r_{c}$, as $\int_{0}^{r_{c}} 2 \pi r \exp (-r / a) \mathrm{d} r$ and $\int_{r_{c}}^{\infty} 2 \pi r K_{\Phi} \exp \left(-r / \xi_{4}\right) \mathrm{d} r$. The quantity $K_{\Phi}$ was found to depend on volume fraction, as $K_{\Phi=0.66}=0.0105 \pm 0.0016$ and $K_{\Phi=0.64}=0.0063 \pm$ 0.0015. For $\Phi_{\text {eff }}=0.66$, we calculate that the $r<r_{c}$ part of the integral in Eq. (4) contributes about $1 \%$ to the entire integral, while for $\Phi_{\text {eff }}=0.64$, it represents more than $25 \%$ of the entire integral. Even though this short distance contribution which pollutes $\chi_{4}$ vanishes at large volume fraction, it makes it risky to use $\chi_{4}$ for processing experimental results. Indeed, in an experiment, the importance of this contribution cannot be estimated without measuring $G_{4}$ explicitly.
Another important source of error arises from the volume fraction dependence of the prefactor $A$ in Eq. (5). Using Eq. (8) with $p=0$, Eq. (5) writes:

$$
2 \pi \rho \xi_{4}^{2}\left(\tau^{\star}\right) \sim \frac{\chi_{4}\left(\tau^{\star}\right)}{K_{\Phi} G_{4}\left(0, \tau^{\star}\right)}
$$

with $K_{\Phi} G_{4}\left(0, \tau^{\star}(\Phi)\right)=A\left(\tau^{\star}\right)$. The quantity $G_{4}(0, \tau)$, displayed in Figure 5, is found to decrease with the lag time $\tau$ and to depend on volume fraction. We find that $K_{0.64} G_{4}\left(0, \tau^{\star}(0.64)\right)=0.0011$ at $\Phi_{\text {eff }}=0.64$ and $K_{0.66} G_{4}\left(0, \tau^{\star}(0.66)\right)=0.0020$ at $\Phi_{\text {eff }}=0.66$. Since the prefactor $A\left(\tau^{\star}\right)$ depends significantly on volume fraction, we can not obviously estimate the growth of $\xi_{4}$ with volume fraction, using $\chi_{4}\left(\tau^{\star}\right)$ alone.

\section{Conclusion}

In conclusion, with the standard direct tool $G_{4}$, we measure the growth of a dynamical correlation length $\xi_{4}$ from 1 to $93 \pm 63$ effective particle radii with increasing volume fraction in our soft microgel suspension. This shows that the dynamics become highly heterogeneous in space when approaching the glass transition, consistent with the broad theoretical picture 42. Recently, various experimental studies have focused on the dynamical susceptibility $\chi_{4}$ as a convenient indirect tool to quantify DHs, mainly because the correlation function $G_{4}$ requires finer measurements and data processing. Meanwhile, in various theoretical works, $\chi_{4}$ was understood to be not necessarily reliable [18, 33, 43, 44].

Our results provide the first quantitative experimental evidence that there is a significant error in estimating the dynamic correlation length $\xi_{4}$ through $\chi_{4}$. The origin of the failure of $\chi_{4}$ is related to the fact that (i) it contains the correlations at the scale of the particle radius and (ii) the prefactor $A(\tau)$ in Eq. (5) varies with volume fraction. It implies that $\chi_{4}$ should a priori never be used as a quantitative indicator of the increase of the spatial correlations, before having first performed a direct measurement of $G_{4}$ to estimate its validity. Nevertheless, as it is the variance of the time correlation function $Q_{t}(\tau)$, the susceptibility $\chi_{4}$ is expected to remain an indicator of the lag time for which the dynamics is the most heterogeneous. In our experiments, the peak occurs approximately at the suspension relaxation time $\tau^{\star}$ defined by $Q\left(\tau^{\star}\right)=0.25$, see Fig. 3 .

The two sources of error highlighted in this work should be kept in mind when trying to relate the dynamic heterogeneities with other features of the glass transition, such as e.g. the local structure [45, 46] or the soft modes of vibration [47, 48. They should be investigated in other glass forming materials and using other observable quantities. 
Acknowledgements

We thank E. Bertin, L. Cipelletti, O. Dauchot, J.-B. Fournier, F. van Wijland and L. Wilson for fruitful discussions, and A. Callan-Jones for a critical reading of the manuscript. We acknowledge support from PICS-CNRS SofTher (B.A. (P.I.), R.C. and A.A.).

\section{APPENDIX}

Image pre-processing: The images are preprocessed in 4 steps. We divide first the images by an average blank image taken with a water only sample to account for dust on the camera and spatial inhomogeneity in the illumination. The second step is an optional correction of the drift of the sample relative to the objective. We use the beads embedded in the suspension, which primarily are used for computing the volume fraction at $30^{\circ} \mathrm{C}$, to record the motion of the sample. Usually 2 to 3 beads are visible in the entire field of view of the CCD camera. We compute the average velocity of the centre of mass of the beads, smoothed over 1000 frames using a temporal sliding window. A subset of the image, as large as possible to fit in the field of view while being advected is extracted by advecting the subframe at the drift speed, using a bilinear pixel interpolation to extract pixel values. Further preprocessing consists in applying 2 filters. A rolling ball background subtraction (radius 60 px) to eliminate heterogeneities in illumination coming from out-of-focus beads, and a Savitzky and Golay filter (local polynomial interpolation, width $=5 \mathrm{px}$, order $=4$ ) is applied to reduce the effect of the camera shot noise.
[1] C. Angell, Science, 1995, 267, 1924.

[2] L. Berthier and G. Biroli, Reviews of Modern Physics, 2011, 83, 587-645.

[3] A. J. Liu and S. R. Nagel, Annual Review of Condensed Matter Physics, 2010, 1, 347-369.

[4] P. N. Pusey and W. van Megen, Nature, 1986, 320, 340342.

[5] E. R. Weeks, J. C. Crocker, A. C. Levitt, A. Schofield and D. A. Weitz, Science, 2000, 287, 627.

[6] W. K. Kegel and A. van Blaaderen, Science, 2000, 287, 290.

[7] M. Ediger, Annual Review of Physical Chemistry, 2000, 51, 99-128.

[8] R. Richert, Journal of Physics : Condensed Matter, 2002, 14, R703-R738.

[9] H. Sillescu, Journ. of Non-crystalline Solids, 1999, 243, 81-108.

[10] A. H. Marcus, J. Schofield and S. Rice, Phys. Rev. E, $1999,60,5725$.

[11] N. Lacevic, F. W. Starr, T. B. Schrøder and S. C. Glotzer, Journal of Chemical Physics, 2003, 119, 7372-7387.

[12] P. Wang, C. Song and H. A. Makse, Nature Physics, 2006, $526-531$.

[13] P. Chaudhuri, W. Kob and L. Berthier, Phys. Rev. Lett., 2007, 99, 060604.

[14] R. Colin, A. M. Alsayed, J.-C. Castaing, R. Goyal, L. Hough and B. Abou, Soft Matter, 2011, 7, 4504-4514.

[15] G. Biroli and J. P. Bouchaud, Structural Glasses and Supercooled Liquids: Theory, Experiment, and Applications, John Wiley \& Sons, Inc., Hoboken, NJ, USA., 2012.

[16] L. O. Hedges, R. L. Jack, J. P. Garrahan and D. Chandler, Science, 2009, 323, 1309.

[17] L. Berthier, Phys. Rev. E, 2004, 69, 020201.

[18] L. Berthier, G. Biroli, J.-P. Bouchaud and R. L. Jack, Overview of different characterisations of dynamic heterogeneity in "Dynamical Heterogeneities in Glasses, Colloids, and Granular Media", Oxford University Press, 2011.

[19] C. Dasgupta, A. V. Indrani, S. Ramaswamy and M. K. Phani, Europhys. Lett., 1991, 15, 307-312.
[20] C. Donati, S. Franz, S. C. Glotzer and G. Parisi, Journal of Non-Crystalline Solids, 2002, 307-310, 215-224.

[21] M. Vogel and S. C. Glotzer, Phys. Rev. E, 2004, 70, 061504 .

[22] D. Chandler, J. P. Garrahan, R. L. Jack, L. Maibaum and A. C. Pan, Phys. Rev. E, 2006, 74, 051501.

[23] A. Parsaeian and H. E. Castillo, Phys. Rev. E, 2008, 78, 060105.

[24] L. Berthier, G. Biroli, J.-P. Bouchaud, L. Cipelletti, D. E. Masri, D. L'Hôte, F. Ladieu and M. Pierno, Science, 2005, 310, 1797-1800.

[25] K. N. Nordstrom, J. P. Gollub and D. J. Durian, Phys. Rev. E, 2011, 84, 021403.

[26] P. Ballesta, A. Duri and L. Cipelletti, Nature Phys., 2008, 4, 550-554.

[27] A. R. Abate and D. J. Durian, Phys. Rev. E, 2007, 76, 021306.

[28] A. Duri and L. Cipelletti, Europhys. Lett., 2006, 76, 972978.

[29] D. Sessoms, I. Bischofberger, L. Cipelletti and V. Trappe, Phil. Trans. R. Soc. A, 2009, 367, $5013-5032$.

[30] E. R. Weeks, J. C. Crocker and D. A. Weitz, Journal of Physics : Condensed Matter, 2007, 19, 205131.

[31] T. Narumi, S. V. Franklin, K. W. Desmond, M. Tokuyama and E. R. Weeks, Soft Matter, 2011, 7, 1472.

[32] F. Lechenault, O. Dauchot, G. Biroli and J. Bouchaud, Europhys. Lett., 2008, 83, 46002.

[33] O. Dauchot, D. Durian and M. van Hecke, Dynamical Heterogeneities in grains and foams in "Dynamical Heterogeneities in Glasses, Colloids, and Granular Media", Oxford University Press, 2011.

[34] C. Crauste-Thibierge, C. Brun, F. Ladieu, D. L'Hôte, G. Biroli and J.-P. Bouchaud, Phys. Rev. Lett., 2010, 104, 165703.

[35] D. El Masri, L. Berthier and L. Cipelletti, Phys. Rev. E, 2010, 82, 031503.

[36] B. R. Saunders and B. Vincent, Advances in Colloid and Interface Science, 1999, 80, 1-25.

[37] Microgel Suspensions: Fundamentals and Applications, ed. A. Fernandez-Nieves, H. M. Wyss, J. Mattsson and 
D. A. Weitz, 2011

[38] H. Senff and W. Richtering, The Journal of Chemical Physics, 1999, 111, 1705-1711.

[39] W. S. Rasband, ImageJ, U. S. National Institutes of Health, Bethesda, Maryland, USA, http://imagej.nih.gov/ij/, 1997-2011.

[40] A. Duri, D. A. Sessoms, V. Trappe and L. Cipelletti, Phys. Rev. Lett., 2009, 102, 085702.

[41] L. Cipelletti, H. Bissig, V. Trappe, P. Ballesta and S. Mazoyer, Journal of Physics: Condensed Matter, 2003, 15, $\mathrm{S} 257$.

[42] L. Berthier, G. Biroli, J.-P. Bouchaud, L. Cipelletti and W. Van Saarloos, Dynamical Heterogeneities in Glasses, Colloids, and Granular Media, Oxford University Press, 2011.

[43] R. Pastore, M. Pica Ciamarra, A. de Candia and A. Coniglio, Phys. Rev. Lett., 2011, 107, 065703.

[44] O. Takeshi, S. Goto, T. Matsumoto, A. Nakahara and M. Otsuki, Phys. Rev. E, 2013, 88, 062108.

[45] C. P. Royal and S. R. Williams, Physics Report, 2015, 560, 1-75.

[46] S. Karmakar, C. Dasgupta and S. Sastry, Annu. Rev.
Cond. Matt. Phys., 2014, 5, 255-284.

[47] K. Chen, M. L. Manning, P. J. Yunker, W. G. Ellenbroek, Z. Zhang, A. J. Liu and Y. A. G., Phys. Rev. Lett., 2011, 107, 108301.

[48] A. Widmer-Cooper, H. Perry, P. Harrowell and D. R. Reichman, Nature Physics, 2008, 4, 711-715.

[49] The decay time $\tau^{\star}$ can be arbitrarily defined as $Q\left(\tau^{\star}\right)=$ 0.25 . In the range $0.2<Q(\tau)<0.4$, the curves of $Q$ are parallel to each other and the increase in $\tau^{\star}$ with volume fraction does not depend much on the value of $Q$.

[50] To perform the fits, the weight for each $G_{4}\left(r, \tau^{\star}\right)$ data point was chosen as the number of pairs of ROIs involved in the average over the whole sample $(17 \times 17$ ROIs $)$, see Eq. (1).

[51] Noise levels equal to $0.3,1$ and 3 standard deviation of the original pixel intensity distribution were added. At 0.3 and 1 standard deviations, the resulting normalised $G_{4}\left(r, \tau^{\star}\right)$ profiles (not shown) were unnoticeably altered and the value of $\xi_{4}$ was correspondingly unaffected. Only at a noise level of 3 standard deviations was the first spatial decay of $G_{4}$ substantially stronger. 